\title{
Some Aspects of the Problem of Progressive Development of Clinical Immunology
}

ISSN: 2637-773X

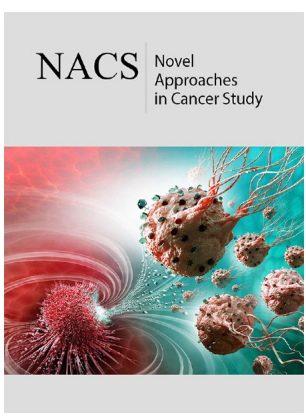

*Corresponding author: Zemskov VM, AV Vishnevsky National Medical Research Center of Surgery, Moscow, Russia

Submission: 湝: October 07, 2020

Published: 眥 November 02, 2020

Volume 5 - Issue 3

How to cite this article: Zemskov VM, Neymann V, Pronko KN, Zemskov AM. Some Aspects of the Problem of Progressive Development of Clinical Immunology. Nov Appro in Can Study. 5(3). NACS.000613. 2020 DOI: 10.31031/NACS.2020.05.000613

Copyright@ Zemskov VM, This article is distributed under the terms of the Creative Commons Attribution 4.0 International License, which permits unrestricted use and redistribution provided that the original author and source are credited.

\author{
Zemskov VM${ }^{1 *}$, Neymann $\mathrm{V}^{2}$, Pronko $\mathrm{KN}^{3}$ and Zemskov $\mathrm{AM}^{4}$ \\ ${ }^{1} \mathrm{AV}$ Vishnevsky National Medical Research Center of Surgery, Russia \\ ${ }^{2}$ VRFD SA, Switzerland \\ ${ }^{3}$ Facecontrol Systems, Moscow, Russia \\ ${ }^{4}$ Voronezh State Medical University, Voronezh, Russia
}

\begin{abstract}
The problems that stand in the way of successful progressive development of clinical immunology that must be overcome. However, this problem is quite complex and contradictory due to the emergence of a new fourth, previously not considered mechanism for the development of almost any pathological conditions, consisting in the development of immune disorders that are interconnected with various body systems and are in reciprocal interaction with them.
\end{abstract}

Keywords: Clinical immunology; Immunoregulation; Immunodiciency; Immunocorrection

\section{Introduction}

It should be noted that the situation of progressive development of clinical immunology is currently quite complex and contradictory. The reason is the establishment of a new, fourth mechanism for the development of almost any, especially chronic diseases, i.e. immune disorders (deficiency states, auto aggressive reactions, hypersensitivity), as factors of their severity, as well as the formation of the necessary attributes of a new medical discipline, since the corresponding specialty has been approved, a network of medical institutions, scientific journals has been created, the production of a wide range of immunotropic drugs has been established, etc.).

At the same time, teaching has been carried out for more than a dozen years in medical, pharmaceutical and other universities of immunology, allergology with the training of relevant specialists. Finally, it should be noted the accumulation of a significant layer of immunologically compromised individuals with inadequately stimulated or suppressed defense reactions that increase the risk of developing infectious, malignant, autoimmune and other diseases in the population as a whole, reducing the reproductive capabilities of the organism and contributing to the appearance of inferior offspring, etc., which requires immunoassay of therapeutic and preventive measures on a large scale.

It should be recognized that, despite the fairly frequent use of modulators of defense reactions in practical medicine, in general, the effectiveness of immunoreaction is rather low and therefore has not become a widespread method of treatment. Perhaps this is due to the fact that clinical immunology has not yet created a well-founded concept or ideology of directed regulation of the perverse function of the immune system. To this should be added a change in the interpretation of some axioms of clinical immunology. For example, the prevailing ideas about fixed (typical) changes in immune reactivity in specific diseases and the stability of targets of modulators, regardless of any circumstances, no longer correspond to reality. Clinical immunologists are faced with the facts of a high qualitative and quantitative modification of the nature of changes in defense reactions in the same pathological processes, but in different patients, and equally pronounced variations in the effects of modulators in various diseases, depending on their clinical and other characteristics. The consequence of this, in some cases, was the unreasonable or arbitrary prescription of modulators to patients. At the same time, a generally predictable directed (targeted) effect on the immune system is quite difficult, since the latter is inertial, in a certain sense conservative, and with excessive 
stimulation or suppression, it can induce mechanisms of selfdestruction, loss of censor function, etc.

The aforementioned phenomenon is based on the following mechanisms.

1. Hierarchical complexity of the structure, i.e. division into central and peripheral organs of immunogenesis, i.e. anatomical fragmentation of the system.

2. Morphological multicomponent of immune responses, consisting in the presence of populations and subpopulations of lymphoid, auxiliary, and other cells.

3. Multilevel regulation of immune reactivity (anti-infectious resistance) by cellular, humoral (antibody, cytokine, hormonal, complementary), nervous and other mechanisms, nucleic acids, metabolic factors, etc.

4. Immunotropic (stimulating and suppressive) effect of traditional drugs, non-drug effects.

5. Achieving homeostasis in various ways through activation/ inhibition of suppressive, other immune and non-immune reactions, modification of the number and function of regulatory populations and subpopulations of lymphocytes.

6. Influence on the immune reactivity of sex, age of the examined persons, menopause, pregnancy, circadian, circannual and other biological rhythms.

7. The likelihood of alternative changes in the components of the immune status in different patients with the same pathological process.

8. The presence of a general organism effect in immunotropic drugs.
9. Quantitative and qualitative variations in immune parameters in patients depending on the pathogenesis, combination, localization, stage, allergization and other features of the disease.

10. The qualitative difference in the state of anti-infectious and other resistance in healthy, recovered, vaccinated, stimulated, suppressed by various drugs contingents.

11. The presence of evolutionarily developed and exclusive, including surgical, ways to achieve balance in the immune system.

12. Genetic control of all the above mechanisms by IR genes, their products, antigens of the $\mathrm{ABO}$ system, Rh factor, haptoglobin, etc. Insufficient consideration of these and other phenomena reduces the overall effectiveness of targeted correction of immunopathology, as a result of which this promising method of treatment did not become widespread. To this should be added methodological errors in the planning and testing of new methods of diagnostics and treatment of patients, mathematical analysis of the results obtained, etc.

At the same time, the basic principles of new diagnostic, therapeutic, preventive innovative technologies have been developed, which should be included in medical standards and used in everyday activities by practitioners of any profile. Thus, in order to achieve the real goal of eliminating immunopathology, it is required to reproduce a whole range of effects on the patient, and to do this deliberately on the basis of correctly conducted clinical and laboratory studies. The expediency of using the relevant information from the literature is also not excluded. 